\title{
The effects of the force of contraction and elbow joint angle on mean and median frequency analysis for muscle fatigue evaluation
}

\author{
Sirinee Thongpanja ${ }^{\mathrm{a}}$, Angkoon Phinyomark ${ }^{\mathrm{a}, \mathrm{b}, *}$, Huosheng $\mathrm{Hu}^{\mathrm{c}}$, Chusak Limsakul ${ }^{\mathrm{a}}$, \\ Pornchai Phukpattaranont ${ }^{\mathrm{a}}$ \\ a Department of Electrical Engineering, Faculty of Engineering, Prince of Songkla University, \\ 15 Kanjanavanich Road, Kho Hong, Hat Yai, Songkhla 90112 Thailand \\ b Faculty of Kinesiology, University of Calgary, Canada \\ c School of Computer Science \& Electronic Engineering, University of Essex, UK
}

*Corresponding author, e-mail: angkoon.p@hotmail.com, aphinyom@ucalgary.ca

Received 28 Jun 2014

Accepted 25 Aug 2015

\begin{abstract}
Mean frequency (MNF) and median frequency (MDF) are often used to assess muscular fatigue from surface electromyography (sEMG) signals. To determine muscle fatigue at different levels of isometric and dynamic contractions, it is necessary to know the relationship between the force of contraction and the MNF/MDF methods. Despite numerous studies, no unique answer has been found. This study re-examines this relationship by investigating 19 subjects and performing isometric and dynamic contractions over a full elbow range of motion measured from the biceps brachii muscle. We obtained the following key results: (i) a strong positive relationship between MNF/MDF methods and the force of contraction was found frequently during isometric contractions at an angle less than $90^{\circ}$ and during dynamic contractions in 3 subjects (16\%); (ii) a strong negative relationship during isometric contractions at an angle greater than $90^{\circ}$ and during dynamic contractions in 8 subjects (42\%), and (iii) no strong linear relationship during isometric contractions for all the angles and during dynamic contractions in 8 subjects (42\%). After possible causes for these conflicting results are discussed, the study then investigates if it is possible to use anthropometric variables of subjects as the criteria to create homogeneous subgroups of relationships. A number of anthropometric variables were used to separate the subgroups, i.e., the forearm and biceps circumferences for males and the hand and shoulder breadths for females.
\end{abstract}

KEYWORDS: anthropometric variables, electromyography signal, feature extraction, spectral analysis

\section{INTRODUCTION}

Muscle fatigue is generally defined as an activityinduced loss of the ability to produce force with a muscle or a muscle group ${ }^{1}$. It is commonly caused by prolonged or repetitive actions. Apart from discomfort and pain, long-time exposure to muscle fatigue can cause injury to the subject, which is often irreversible. If an automated fatigue identification system were feasible, it could be used as an indicator to reduce the chances of workplace injury ${ }^{2}$. To develop an automated fatigue identification system, a number of sources and techniques can be used to objectively determine the level of muscle fatigue in a subject, e.g., force gauges, sonomyography, nearinfrared spectroscopy, mechanomyography, acoustic myography, electroencephalography, and surface electromyography (sEMG) signals $^{3}$.

The sEMG signals have been widely applied in the field of motion pattern recognition, control ${ }^{4,5}$, and muscle fatigue analysis. The sEMG signals use non-invasive procedures and are capable of monitoring a particular muscle in real-time ${ }^{6}$. Moreover, the assessment of human muscle fatigue from sEMG signals can be applied in a wide class of situations, including the low back pain in helicopter pilots ${ }^{7}$, muscle fatigue in children with cerebral palsy ${ }^{8}$, muscle fatigue during playing games ${ }^{9}$, and muscle fatigue during repeated cycling sprints ${ }^{10}$. Several classical and modern signal processing methods have been used as a relative indicator of the muscle fatigue phenomenon for an individual subject such as the root mean square, zero crossing rate, averaged instantaneous frequency, wavelet analysis, 
fractal analysis, and mean frequency (MNF), and median frequency (MDF) ${ }^{11}$.

Since the muscle fatigue results in a downward shift of the EMG frequency spectrum, it can be tracked by monitoring some statistical characteristics of the spectrum, i.e., mean and median. Until now, the MNF and MDF methods have been effectively applied to the sEMG signals as a mean of identifying muscle fatigue ${ }^{12}$, and become the gold standard for assessment of muscle fatigue. It is commonly accepted in the literature that MNF and MDF methods are reliable parameters and efficient ways to estimate muscle fatigue during either isometric or dynamic muscle contraction ${ }^{13}$.

There is a large variance however in both the sEMG power spectrum itself and a nature of the power spectrum shift as a result of fatigue onset for different subjects in general. Moreover, daily activities involve dynamic muscle contractions (as muscle force and/or geometry are changing) that yield nonstationary sEMG signals ${ }^{13}$. For these reasons, it is difficult to use this information to reliably automate the process of fatigue onset determination without the knowledge of the effects of time-varying factors on $\mathrm{MNF}$ and $\mathrm{MDF}^{12}$.

The individual effects of these factors on the MNF and MDF methods have been investigated in previous studies ${ }^{14,15}$. Based on the literature, three main time-varying factors should be considered: (1) the number and firing rate of active motor units (MUs), (2) muscle geometry based on muscle length or joint angle, and (3) muscle force based on the force of contraction ${ }^{15}$.

Effects of the first two factors on MNF and MDF have been conclusive. For the first factor, the firing statistics of active MUs are the main factors that affect the power spectrum of the sEMG signals based on a widely accepted model and supported by the experimental evidence ${ }^{16}$. The effect of the firing rate, however, appears only in the low frequency range $(0-40 \mathrm{~Hz})$, so the MNF and MDF methods are weakly dependent on the firing rate ${ }^{16,17}$. Previous theoretical ${ }^{17,18}$ and experimental ${ }^{19}$ studies report that although the firing statistics of active MUs were changed, they do not significantly affect the MNF and MDF.

On the other hand, the shapes of the motor unit action potentials (MUAPs) affect the power spectrum of the sEMG signals ${ }^{16}$. The effects of the MUAP shape appear beyond $40 \mathrm{~Hz}$ power spectrum, so time-dependent MUAP waveforms may generate corresponding changes in MNF and MDF. Variations in the shapes of MUAPs can result from changes in two components: (1) muscle geometry consisting of fibre diameter, electrode configuration and subcutaneous tissue thickness, and (2) muscle force during the recruitment of MUs of different types or locations. Further, the muscle geometry (the second factor) resulting from changes in joint angle does significantly affect MNF and MDF ${ }^{15}$. Most previous studies report that MNF and MDF values decreased with increasing muscle length (i.e., decreasing elbow joint angle in degrees of flexion) for various muscles including the biceps brachii muscle ${ }^{20-23}$.

For the third factor, however, a general consensus on the effect of the muscle's force of contraction on the MNF and MDF methods has yet to be reached ${ }^{15,24}$. The reported conflicting results can be categorized into three cases: (1) No linear relationship: MNF and MDF values are weakly affected or unaffected by changes in the levels of contraction ${ }^{15}$, (2) a strong positive linear relationship: MNF and MDF values increase as muscle contraction levels increase ${ }^{14}$, and (3) a strong negative linear relationship: MNF and MDF values decrease as muscle contraction levels increase ${ }^{24}$. A better understanding of whether interactions exist between the force of contraction and joint angle, which may affect the MNF and MDF values, is warranted.

The first purpose of this study is therefore to re-examine the effect of the force of contraction on MNF and MDF of the sEMG signals during isometric and dynamic contractions at different joint angles. Based on the results from previous studies, it was hypothesized that all the three cases mentioned above exist and may depend on the force of contraction, joint angle, and individual subjects. To the best of our knowledge, an investigation of this effect from sEMG signals measured from a sample of both male and female healthy subjects from both isometric and dynamic contractions at different joint angles has never been completed (Table 1). Further, possible causes for these conflicting results are also discussed.

Individual differences in the sEMG signals have been found, which may be due to human physical variations 39,40 . The second purpose of this study was therefore to investigate the feasibility to use twelve related anthropometric variables of subjects as the criteria to identify homogeneous subgroups related to three relationship cases. Knowing this relationship would be useful for designing an appropriate automated fatigue identification system which can adapt to the sEMG signals that varies from person to person during different levels of muscle contraction and joint angles. The analysis 
Table 1 A summary of the experimental conditions in the previous investigations on muscle effect on MNF and MDF in chronological order.

\begin{tabular}{lrrccccccc}
\hline Year & Ref. & $N^{\dagger}$ & Age & Muscle $^{*}$ & IED $^{\dagger}$ & Force levels & RT $^{\dagger}$ & Filtering & Case \\
\hline 1978 & 25 & 7 & - & RF, VL, VM & - & - & - & - & I \\
1980 & 26 & 8 & $22-52$ & FCR & 40 & $5-100 \%$ MVC & 3 & - & I \\
1980 & 27 & 10 & $23.2 \pm 2.3$ & BR & 40 & $10,20,40,60,80,100 \%$ MVC & 3 & - & I \\
1982 & 28 & 4 & $21-24$ & BB, BC, BR & 20 & $5,10,15,20,25,30,40,50,80 \%$ MVC & $3-5$ & $0.2-2000$ & I, II \\
1982 & 29 & 5 & $32.5 \pm 8.2$ & BB & - & $0.25,0.5,1,2,3 \mathrm{~kg}$ & 10 & - & II \\
1984 & 30 & 26 & $26.6 \pm 6.4$ & FDI & 10 & $20,80 \%$ MVC & $3-5$ & $30-350$ & I \\
1984 & 31 & 19 & $18-32$ & FT, CS & 15 & $20,40,60,80 \%$ maximum amplitude & 3 & $3-520$ & II \\
1985 & 32 & 6 & $20-40$ & BB & - & $1-10$ Nm & 8.2 & - & II \\
1986 & 33 & 9 & $30-40$ & BB, ED & 35 & $30,50,70,90 \%$ MVC & 6 & $1-1000$ & I \\
1987 & 34 & 12 & $26.3 \pm 2.5$ & BB & 6 & $0-80 \%$ MVC & 5 & $<520$ & II \\
1989 & 35 & 14 & $36 \pm 8$ & TZ & 30 & $0-100 \%$ MVC & $10-15$ & $5-500$ & II \\
1990 & 36 & 9 & $30-40$ & BB & - & $20,40,60,80,100 \%$ MVC & $1-2$ & - & II \\
1991 & 37 & 14 & $30.2 \pm 7.8$ & TB, AN & 6 & $10,20,40,60,80 \%$ MVC & 3 & $16-800$ & I \\
1999 & 38 & 10 & $30.2 \pm 6.1$ & BB & 10 & $10,30,50,70 \%$ MVC & 30 & $10-450$ & III \\
2001 & 15 & 12 & $31.1 \pm 10$ & BB & 40 & $20,30,40,50,60 \%$ MVC & 5 & $0.1-3000$ & I \\
2008 & 14 & 12 & $24.8 \pm 2.8$ & BB, TB, BR & 10 & $10,20,30,40,50,60,70 \%$ MVC & 8 & $20-450$ & II \\
2009 & 24 & 94 & $5-69$ & BB & 10 & $10,30,50,70,100 \%$ MVC & 5 & $20-500$ & III \\
& & & & & & & &
\end{tabular}

' $N$ is the number of subjects; IED is inter-electrode distance (mm); RT is recording time (s); '-' is 'not available'.

" $\mathrm{RF}$ is the rectus femoris muscle; VL is the vastus lateralis muscle; VM is the vastus medialis muscle; FCR is the flexor carpi radialis muscle; $\mathrm{BR}$ is the brachioradialis muscle; $\mathrm{BB}$ is the biceps brachii muscle; $\mathrm{BC}$ is the brachialis muscle; FDI is the first dorsal interosseous muscle; FT is the frontalis muscle; CS is the corrugator supercilii muscle; ED is the extensor digitorum muscle; $\mathrm{TZ}$ is the trapezius muscle; $\mathrm{AN}$ is the anconeus muscle; $\mathrm{TB}$ is the triceps brachii muscle.

of the MNF and MDF methods together with anthropometric variables was only previously studied by Suuden et $\mathrm{al}^{41}$. To the best of our knowledge, the investigation of this relationship for studying the effect of the force of contraction on the MNF and MDF methods has never been studied before.

\section{MATERIALS AND METHODS}

\section{Subjects and data acquisition}

Two sets of data were measured and used to investigate the effect of the force of contraction on MNF and $\mathrm{MDF}^{42}$. A total of 19 subjects participated in this study: ten males (21.0 \pm 0.88 years) and nine females (21.11 \pm 0.78 years). All participants had no prior history of neuromuscular disorders and, prior to collecting the data, all participants provide their written informed consent to participate. The first experiment involves isometric muscle contractions while the second experiment involves dynamic muscle contractions at elbow angles between $0^{\circ}$ and $150^{\circ}$. For both experiments, the sEMG signals were recorded from bipolar electrodes (H124SG, Kendal ARBO) placed on the biceps brachii muscle. A pair of disposable pre-gelled self-adhesive $\mathrm{Ag} / \mathrm{AgCl}$ surface electrodes of $24 \mathrm{~mm}$ diameter (circular) was applied to each subject at an inter-electrode distance of $20 \mathrm{~mm}$, after suitable preparation of the skin with alcohol. A multi-channel electrophysiological signal measurement system (Mobi66b, TMS International B.V., Netherlands) with 20$500 \mathrm{~Hz}$ bandwidth, 19.5× gain, and 100-dB CMRR was used to acquire the sEMG data with a sampling rate of $1024 \mathrm{~Hz}$ and a high data resolution of 24 bits.

In the first experiment, the subject was asked to lift a required external load involving $1,2,3,4$, and $5 \mathrm{~kg}$ at a specific elbow joint angle involving $30^{\circ}$, $60^{\circ}, 90^{\circ}, 120^{\circ}$, and $150^{\circ}$ of flexion. It is important to note that the $0^{\circ}$ of extension is when the subject's arm is completely straight. After the subject's elbow joint angle was stable, the sEMG data were recorded for $5 \mathrm{~s}$. A break until the subject was feeling 'flesh' was taken between each measurement to avoid fatigue of the muscle. The order of the loadangle combinations was randomized. Each of the twenty-five load-angle combinations was performed five times per day for four separate days to include the effect of fluctuating sEMG signals between days in the same subject. In total, 9500 five-second data trials (19 subjects $\times 5$ external loads $\times 5$ joint 
angles $\times 5$ trials $\times 4$ days) were collected for further signal analysis.

In the second experiment, the subject was asked to perform an elbow flexion-extension task with a required external load involving $1,2,3,4$, and $5 \mathrm{~kg}$ ranging from a full extension $\left(0^{\circ}\right)$ to a full flexion (approximately $150^{\circ}$ ), for $3 \mathrm{~s}$ in duration. Five trials were performed per day for four separate days, so 1900 three-second data trials ( 19 subjects $\times 5$ external loads $\times 5$ trials $\times 4$ days) were collected in total for further signal analysis.

Finally, twelve anthropometric variables of the subjects were measured consisting of (1) body mass, (2) standing height, (3) biceps circumference, (4) elbow-hand grip length, (5) shoulder breadth, (6) elbow-fingertip length, (7) forearm circumference, (8) forward grip reach, (9) hand breadth, (10) hand length, (11) shoulder-elbow length, and (12) body mass index (BMI), an individual's body mass in kilograms divided by the square of the standing height in meters. Details of all the variable definitions along with details of the measuring instruments can be found in Phinyomark et $\mathrm{al}^{43}$.

\section{Signal analysis}

First, the sEMG signals measured during isometric and dynamic contractions at different external loads were normalized to the peak activation levels obtained during the elbow flexion-extension task at the maximum load for each individual ${ }^{44}$. Specifically, for each trial the sEMG signals were rectified and smoothed using a Butterworth low-pass filter with a cut-off frequency of $6 \mathrm{~Hz}$. This whole trial linear envelope was then divided into 12 segments with a length of 256 samples $(250 \mathrm{~ms}$ ) and the mean amplitude was computed for each segment. The maximum mean amplitude among the segments was determined for each trial and then the average value over all the trials was used to represent the $100 \%$ peak dynamic amplitude (pk-DYN). For both isometric and dynamic conditions, raw EMG signals were normalized as a percentage of the pk-DYN (\% pk-DYN).

Second, the normalized sEMG signals during both isometric and dynamic contractions were divided into several epochs with an epoch length of 512 samples $(500 \mathrm{~ms})$. The sEMG power spectrum $P_{j}$ was computed from each epoch, and then MNF and MDF were calculated using the following definitions:

$$
\mathrm{MNF}=\frac{\sum_{j=1}^{M} f_{j} P_{j}}{\sum_{j=1}^{M} P_{j}},
$$

$$
\sum_{j=1}^{\mathrm{MDF}} P_{j}=\sum_{j=\mathrm{MDF}}^{M} P_{j}=\frac{1}{2} \sum_{j=1}^{M} P_{j},
$$

where $f_{j}$ is a frequency value at a frequency bin $j$ and $M$ is the number of frequency bins. Average value over all the epochs was used as a feature value for each trial.

Third, to determine the degree of linear association between the levels of muscle contraction and the MNF and MDF values, the correlation analysis was deployed. Three relationship cases reported in previous studies were determined using the strength and direction of correlation coefficients. No linear (or a weak linear) relationship "Case I" was determined if a correlation coefficient of $r$ was between -0.67 and $+0.67^{45}$. A strong positive linear relationship "Case II" was determined if $r$ coefficient was between +0.68 and +1.0 , and a strong negative linear relationship "Case III" was determined if $r$ coefficient was between -1.0 and $-0.68^{45}$.

Finally, twelve anthropometric variables were evaluated to determine the utility in separating the subjects associated with the three relationship cases. The statistically significant difference was defined by $p$-value obtained from the ANOVA and effect size (ES) obtained from Cohen's $d$ method $^{46}$. An alpha value of 0.05 was set for all tests. Due to the exploratory nature of the present analysis, trends were considered $0.05<$ alpha $<1.0$. The ES value is usually interpreted as small effect if $d$ was between 0.20 and 0.49 , medium effect if $d$ was between 0.50 and 0.79 , and large effect if $d$ was greater than $0.8^{46}$.

\section{RESULTS AND DISCUSSION}

\section{Measured sEMG signals}

The sEMG signals measured from the biceps brachii muscle during isometric muscle contractions for the 25 different load-angle combinations and during dynamic muscle contractions for the five different loads are shown in Fig. 1. It is apparent from the figure that measured sEMG signals were not greatly contaminated by any kinds of noise including movement artefact, power-line interference, and electric noise from electronic equipment. In addition, an average actual measurement standard deviation (SD) of MNF across all subjects was 3.33, while an average theoretical SD of MNF across all subjects was 3.75. A theoretical SD of MNF was computed by the relationship ${ }^{47} \mathrm{SD}=0.33 \sqrt{\mathrm{MNF} / T}$, where $T$ is a signal length in duration, i.e., $0.5 \mathrm{~s}$. This result confirms that the observed variation of MNF 
(a)

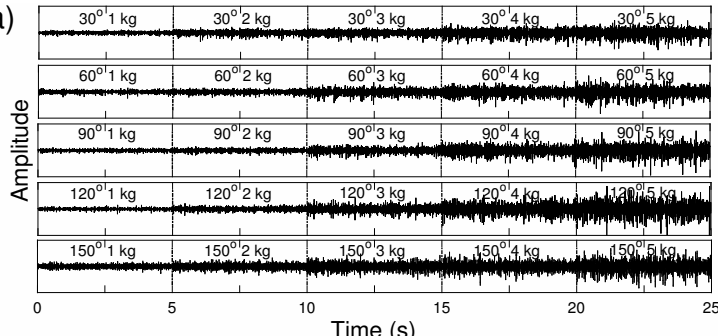

(b)

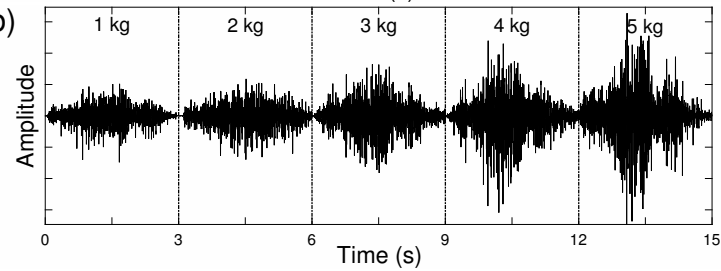

Fig. 1 The sEMG signals in time domain during (a) isometric and (b) dynamic muscle contractions.

and MDF at different levels of muscle contraction in the present investigation were not affected by the measurement error.

\section{Effect of isometric muscle contractions on MNF and MDF}

The first purpose of this study was to re-examine the effect of the force of contraction on MNF and MDF of the sEMG signals during isometric and dynamic contractions at different joint angles. In support of our hypothesis, three relationship cases which have been found in previous investigations ${ }^{14,15,24}$ were also found in the current study (Fig. 2). The current study improves upon prior ones since previous investigations have found only one or two out of the three relationship cases (Table 1), and most previous studies have investigated only the effect of isometric contraction on MNF and MDF (Table 1).

Although there was no consistent trend across elbow joint angles for all the subjects (Table 2), Case I (MNF and MDF values were unaffected or only weakly affected by changes in the levels of contraction) was found through the total range of motion of an elbow joint (Table 2). Conversely, Case II (MNF and MDF values increased with increasing the levels of contraction) was found frequently at an elbow angle less than $90^{\circ}$. Case III (MNF and MDF values decreased with increasing the levels of contraction) was found frequently at an elbow angle greater than $90^{\circ}$ (Table 2). As is evident from the results in this table, it is important to note that if the effect of the contraction force on MNF and MDF was examined based on an average value across all the subjects (as implemented in several studies in
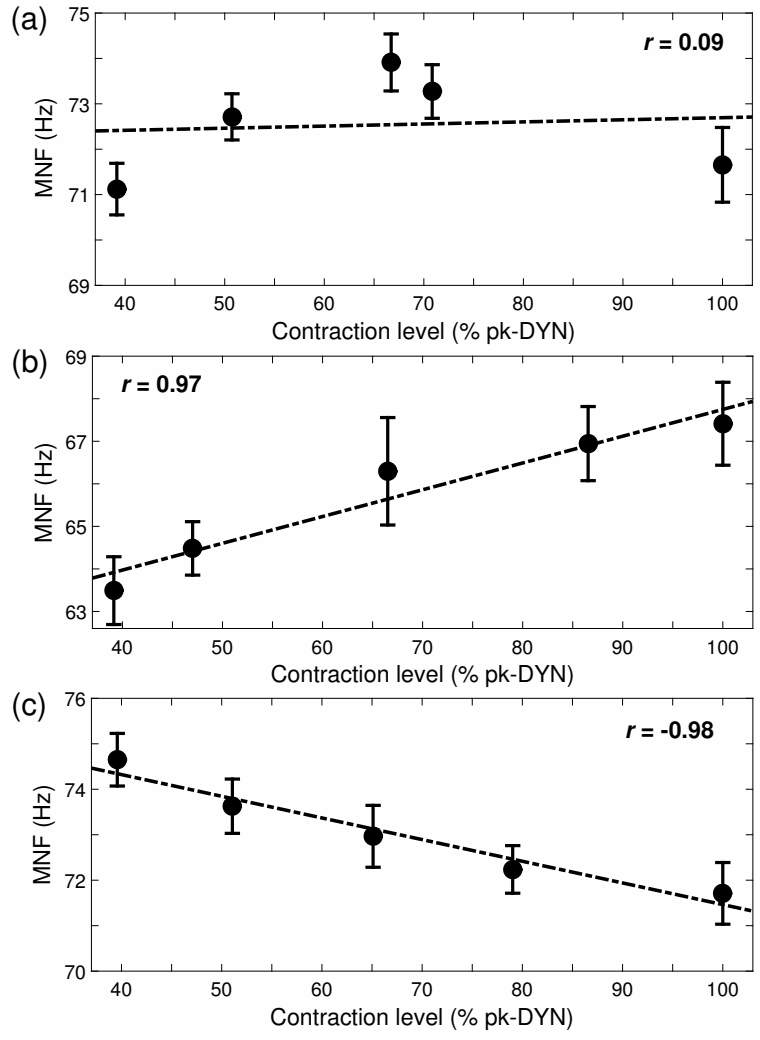

Fig. 2 The relationship between the muscle contraction levels and the MNF values (a) Case I, (b) Case II, (c) Case III. The error bars shown are given by $\mathrm{SD} / \sqrt{n_{\mathrm{et}}}$, where SD is the standard deviation of MNF at a constant angle and $n_{\mathrm{et}}$ is the number of epochs and trials.

Table 1), MNF and MDF values would be unaffected by changes in the levels of contraction across all the angles (Case I).

The results of the present investigation demonstrate that the effect of the force of contraction on MNF and MDF was dependent on the joint angles and, consequently, it is strongly recommended that the levels of muscle contraction and the joint angles be considered together when trying to reduce the muscle effects on the MNF and MDF methods for research purposes.

\section{Effect of dynamic muscle contractions on MNF and MDF}

In support of the first hypothesis, and consistent with the results of isometric muscle contraction, the three relationship cases were found (Table 3 and Table 4). MNF and MDF features extracted from dynamic sEMG signals could be considered as the average value of the MNF and MDF features ex- 
Table 2 The number of subjects for the three relationship cases on the effect of the force of contraction on MNF and MDF extracted from sEMG signals during isometric muscle contractions at five elbow joint angles (degrees of flexion).

\begin{tabular}{|c|c|c|c|c|c|c|c|c|c|c|}
\hline \multirow[t]{2}{*}{ Relationship cases } & \multicolumn{5}{|c|}{ MNF } & \multicolumn{5}{|c|}{ MDF } \\
\hline & $30^{\circ}$ & $60^{\circ}$ & $90^{\circ}$ & $120^{\circ}$ & $150^{\circ}$ & $30^{\circ}$ & $60^{\circ}$ & $90^{\circ}$ & $120^{\circ}$ & $150^{\circ}$ \\
\hline Case I: No linear relationship & 9 & 6 & 7 & 6 & 3 & 6 & 4 & 8 & 7 & 7 \\
\hline Case II: A strong positive linear relationship & 7 & 10 & 6 & 1 & 0 & 10 & 12 & 7 & 2 & 0 \\
\hline Case III: A strong negative linear relationship & 3 & 3 & 6 & 12 & 16 & 3 & 3 & 4 & 10 & 12 \\
\hline
\end{tabular}

Table 3 A summary of the twelve selected anthropometric variables (mean and standard deviation) for the three relationship cases for male group and their statistical analysis.

\begin{tabular}{|c|c|c|c|c|c|c|c|c|c|c|}
\hline \multirow[t]{2}{*}{ Anthropometric variables } & \multicolumn{2}{|c|}{ Case I $(n=5)$} & \multicolumn{2}{|c|}{ Case II $(n=1)$} & \multicolumn{2}{|c|}{ Case III $(n=4)$} & \multirow{2}{*}{$\begin{array}{c}\mathrm{I} \times \mathrm{II} \\
(\mathrm{ES} d)\end{array}$} & \multirow{2}{*}{$\begin{array}{l}\mathrm{I} \times \mathrm{III} \\
(\mathrm{ES} d)\end{array}$} & \multirow{2}{*}{$\begin{array}{l}\mathrm{II} \times \mathrm{III} \\
(\mathrm{ES} d)\end{array}$} & \multirow{2}{*}{$\begin{array}{l}\mathrm{I} \times \mathrm{II} \times \mathrm{III} \\
(p \text {-value })\end{array}$} \\
\hline & Mean & (SD) & Mean & (SD) & Mean & (SD) & & & & \\
\hline Body mass (kg) & 58.2 & (4.6) & 73.0 & $(0.0)$ & 57.5 & $(6.2)$ & $3.94^{* * a}$ & 0.15 & $3.23^{* *}$ & 0.08 \\
\hline Standing height $(\mathrm{cm})$ & 170.4 & (4.9) & 172.0 & $(0.0)$ & 169.6 & (3.9) & 0.40 & 0.19 & $0.79^{*}$ & 0.89 \\
\hline Biceps circumference $(\mathrm{cm})$ & 27.3 & $(2.1)$ & 32.4 & $(0.0)$ & 25.5 & $(1.6)$ & $3.03^{* * \mathrm{~b}}$ & 1.08 & $5.42^{* * \mathrm{a}}$ & 0.04 \\
\hline Elbow-hand grip length $(\mathrm{cm})$ & 36.2 & (1.9) & 39.7 & $(0.0)$ & 35.3 & $(2.2)$ & $2.30^{* * *}$ & $0.50^{*}$ & $2.64^{* * *}$ & 0.21 \\
\hline Shoulder breadth $(\mathrm{cm})$ & 42.4 & (3.8) & 47.3 & $(0.0)$ & 39.9 & $(6.9)$ & $1.59^{* * *}$ & $0.54^{*}$ & $1.40^{* *}$ & 0.48 \\
\hline Elbow-fingertip length $(\mathrm{cm})$ & 48.4 & $(2.5)$ & 52.6 & $(0.0)$ & 47.4 & $(1.7)$ & $2.01^{* *}$ & $0.51^{*}$ & $3.85^{* * \mathrm{~b}}$ & 0.19 \\
\hline Forearm circumference $(\mathrm{cm})$ & 25.1 & (1.7) & 28.2 & $(0.0)$ & 23.7 & $(0.9)$ & $2.30^{* * *}$ & 1.15 & $6.61^{* * \mathrm{a}}$ & 0.06 \\
\hline Forward grip reach $(\mathrm{cm})$ & 79.2 & $(3.1)$ & 80.4 & $(0.0)$ & 79.0 & $(4.0)$ & 0.49 & 0.06 & 0.45 & 0.94 \\
\hline Hand breadth $(\mathrm{cm})$ & 8.9 & $(2.1)$ & 8.9 & $(0.0)$ & 8.7 & $(2.0)$ & 0.01 & 0.10 & 0.13 & 0.99 \\
\hline Hand length (cm) & 17.6 & $(1.6)$ & 18.9 & $(0.0)$ & 18.6 & $(1.1)$ & $0.96^{* *}$ & $0.76^{*}$ & 0.40 & 0.55 \\
\hline Shoulder elbow length $(\mathrm{cm})$ & 37.0 & (1.1) & 38.2 & $(0.0)$ & 37.3 & $(1.7)$ & $1.33^{* * *}$ & 0.19 & $0.68^{*}$ & 0.76 \\
\hline BMI & 20.0 & (1.1) & 24.7 & $(0.0)$ & 20.0 & $(2.0)$ & $5.41^{* * a}$ & 0.04 & $3.01^{* *}$ & 0.07 \\
\hline
\end{tabular}

${ }^{a}$ statistically significant difference $(p<0.05)$.

$\mathrm{b}$ trend towards significant difference $(0.05 \leqslant p<0.10)$.

* represents a medium effect size $(0.5 \leqslant d<0.8)$

** represents a large effect size $(d \geqslant 0.8)$

Table 4 A summary of the twelve selected anthropometric variables (mean and standard deviation) for the three relationship cases for female group and their statistical analysis.

\begin{tabular}{|c|c|c|c|c|c|c|c|c|c|c|}
\hline \multirow[t]{2}{*}{ Anthropometric variables } & \multicolumn{2}{|c|}{ Case I $(n=3)$} & \multicolumn{2}{|c|}{ Case II $(n=2)$} & \multicolumn{2}{|c|}{ Case III $(n=4)$} & \multirow{2}{*}{$\begin{array}{c}\mathrm{I} \times \mathrm{II} \\
(\mathrm{ES} d)\end{array}$} & \multirow{2}{*}{$\begin{array}{l}\mathrm{I} \times \mathrm{III} \\
(\mathrm{ES} d)\end{array}$} & \multirow{2}{*}{$\begin{array}{l}\mathrm{II} \times \mathrm{III} \\
(\mathrm{ES} d)\end{array}$} & \multirow{2}{*}{$\begin{array}{l}\mathrm{I} \times \mathrm{II} \times \mathrm{III} \\
(p \text {-value })\end{array}$} \\
\hline & Mean & (SD) & Mean & (SD) & Mean & (SD) & & & & \\
\hline Body mass (kg) & 51.0 & $(4.6)$ & 51.0 & $(2.8)$ & 47.5 & $(6.4)$ & 0.00 & $0.72^{*}$ & $0.75^{*}$ & 0.65 \\
\hline Standing height $(\mathrm{cm})$ & 160.0 & $(8.9)$ & 159.0 & $(4.2)$ & 156.8 & $(7.3)$ & 0.17 & 0.48 & 0.41 & 0.85 \\
\hline Biceps circumference (cm) & 23.9 & $(1.4)$ & 24.6 & $(4.3)$ & 23.3 & $(2.7)$ & 0.32 & 0.28 & 0.47 & 0.87 \\
\hline Elbow-hand grip length $(\mathrm{cm})$ & 35.5 & $(2.8)$ & 35.5 & $(1.0)$ & 33.9 & $(1.0)$ & 0.02 & $0.97^{* *}$ & 1.96 & 0.47 \\
\hline Shoulder breadth $(\mathrm{cm})$ & 38.7 & $(2.8)$ & 41.1 & $(3.3)$ & 37.4 & $(0.6)$ & $1.02^{* * *}$ & $0.84^{* *}$ & $2.55^{* * \mathrm{~b}}$ & 0.23 \\
\hline Elbow-fingertip length (cm) & 46.5 & $(2.9)$ & 47.1 & $(0.9)$ & 44.4 & $(3.6)$ & 0.31 & $0.76^{*}$ & $1.06^{* *}$ & 0.54 \\
\hline Forearm circumference $(\mathrm{cm})$ & 21.6 & $(1.1)$ & 21.9 & $(1.4)$ & 20.8 & $(1.6)$ & 0.29 & $0.69^{*}$ & $0.85^{* *}$ & 0.63 \\
\hline Forward grip reach $(\mathrm{cm})$ & 67.6 & $(6.0)$ & 65.7 & $(6.9)$ & 71.0 & $(4.7)$ & 0.39 & $0.77^{*}$ & $1.21^{* *}$ & 0.54 \\
\hline Hand breadth $(\mathrm{cm})$ & 7.6 & $(0.4)$ & 7.3 & $(0.1)$ & 6.8 & $(0.3)$ & $1.49^{* * *}$ & $2.98^{* * \mathrm{a}}$ & $2.25^{* * \mathrm{~b}}$ & 0.03 \\
\hline Hand length (cm) & 16.0 & (1.0) & 17.0 & $(0.3)$ & 16.3 & $(1.3)$ & $1.60^{* * *}$ & 0.34 & $0.78^{*}$ & 0.59 \\
\hline Shoulder elbow length $(\mathrm{cm})$ & 36.6 & $(1.5)$ & 36.1 & $(0.1)$ & 34.0 & $(1.0)$ & 0.57 & $2.50^{* * \mathrm{a}}$ & $2.91^{* * a}$ & 0.05 \\
\hline BMI & 20.0 & (1.8) & 20.2 & $(2.2)$ & 19.4 & $(2.4)$ & 0.17 & 0.33 & 0.45 & 0.88 \\
\hline
\end{tabular}

\footnotetext{
${ }^{a}$ statistically significant difference $(p<0.05)$

$\mathrm{b}$ trend towards significant difference $(0.05 \leqslant p<0.10)$.

* represents a medium effect size $(0.5 \leqslant d<0.8)$

** represents a large effect size $(d \geqslant 0.8)$
} 
tracted from isometric sEMG signals across different elbow joint angles. Thus only one case existed for each individual subject for dynamic condition. The results show that Case I (no relationship) was found in 8 subjects ( 5 males and 3 females) or $42 \%$ of the study population. Case II, a positive linear relationship was found in three subjects (1 male and 2 females) or $16 \%$ of the study population. Case III, a negative linear relationship was found in 8 subjects (4 males and 4 females) or $42 \%$ of the study population. Possible causes for the conflicting results found about the effect of the force of contraction during both isometric and dynamic conditions on the MNF and MDF methods are discussed in subsequent sections.

\section{Anthropometric variables}

Based on the results presented above, the linear relationship between the force of contraction and the MNF/MDF value varied from person to person, thus it was hypothesized that it may be due to the human physical variation (i.e., different anatomical and physiological properties of the muscles). Moreover, individual differences in the sEMG signals associated with the anthropometric variables (i.e., the measurement of human individual) have been reported ${ }^{39-41}$. The second purpose of this study was therefore to investigate the feasibility to use one or more of the twelve selected anthropometric variables as the criteria to create homogeneous subgroups of the subjects associated with the three relationship cases.

For each of the twelve anthropometric variables, male and female subjects were divided into one of the three relationship cases (Tables 3 and 4). In support of our hypothesis, a number of anthropometric variables could be used to separate between the subgroups ( $p<0.05$ and $d>0.8)$. Specifically, male subjects with Case I and Case III have smaller biceps and forearm circumferences compared to the male subject with Case II, while Case I male subjects have slightly bigger size of both circumferences compared to Case III male subjects. Other meaningful anthropometric variables for male group are body mass and BMI (I versus II), and elbow-fingertip length (II versus III). For female group, female subjects with Case I have larger hand breadth compared to other subjects while female subjects with Case II have larger shoulder breadth compared to other subjects. In addition, female subjects with Case III have smaller hand and shoulder breadths as well as shorter shoulder elbow length compared to the others.
Since the effect size is directly related to the discrimination being considered, the meaningful anthropometric variables could be possible to use as the criteria to identify homogeneous subgroups in the analysis of both isometric and dynamic muscle contractions using the MNF and MDF methods for research purposes.

\section{Identification and possible causes for the conflicting results}

A summary of the experimental conditions in previous related investigations is presented in Table 1. There are several possible causes for the continuing divergence of results about the effect of the force of contraction on MNF and MDF. These are based in part on results from studies with small population samples ${ }^{28,29,32}$, different demographic groups $^{24,26,28}$, different study designs ${ }^{22}$ involving the levels of muscle contraction, the range of joint angles and the difference in the length of the data measured ${ }^{24,35,36,38}$, the existence of muscle fatigue ${ }^{48,49}$, as well as varying evaluating methods.

A number of causes that may influence the MNF and MDF features were discussed in more detail in the following. The first possible cause is gender- and age-related differences. Bilodeau et $\mathrm{al}^{50}$ reported the difference between male and female subjects for MDF but not for MNF. Kaplanis et $\mathrm{al}^{24}$, on the other hand, detected no significant difference between genders and ages. In the present study, the difference between males and females for MNF and MDF was not directly compared. However, the three relationship cases were found for both genders. While age range of the subjects was very narrow (i.e., 20-23 years), the three relationship cases were also found. Although the effects of gender and age on the MNF and MDF features are still not clear, both factors cannot use as the criteria to create the homogeneous subgroups associated with the effect of the force of contraction.

Interestingly, Bilodeau et $\mathrm{al}^{50}$ reported that the main contributor for gender difference is the skinfold layer. The skinfold measurement test is a common method of determining a body fat percentage and it is one of the anthropometric variables. Although the skinfold layer was not measured in the present study, several measured anthropometric variables are commonly used to estimate the body fat percentage consisting of circumference, body mass, standing height, and BMI. There are a number of formulae that can be used to estimate body fat percentage using anthropometric variables and some of them take gender and age into ac- 
count, such as the formulae proposed by Deurenberg et $\mathrm{al}^{51}$. Future research is needed in this area to better understand the effect of the body fat percentage via the related anthropometric variables involving skinfold, body mass, standing height and $\mathrm{BMI}$ on the MNF and MDF features.

The second possible cause is the muscle studied. Different muscles have different muscle fibre composition and distribution, and different tissue filter effects ${ }^{52}$. Both can produce different spectral changes with the levels of contraction. The differences in fibre diameters and types are also related to the gender difference ${ }^{53}$. Type II fibres have a larger diameter than type I fibres and are recruited later in a contraction, thus the action potential conduction velocity increases with increasing levels of contraction force ${ }^{54}$. This can also produce an increase in the MNF and MDF values. However, the three relationship cases were found in the present study, in which only the biceps brachii muscle was investigated. Moreover, the divergence of the results has also been found in different muscles as is evident from the summary in Table 1 .

The third possible cause is the location of the surface electrodes over the muscle. Komi and Viitasalo ${ }^{55}$ observed an increase in MNF value with the level of contraction force unless the electrodes were located over the motor point area. The fourth possible cause is the variation in the inter-electrode distance of the bipolar surface electrodes. Zipp ${ }^{56}$ reported that the high-pass electrode filter characteristic compensates for the low-pass tissue filter characteristic, and then allowing improved observation of force induced spectral changes. A small interelectrode distance of less than $10 \mathrm{~mm}$ can produce the results which were dependent of force level ${ }^{34}$, while larger inter-electrode distances of equal or more than $20 \mathrm{~mm}$ can give the results which were independent of force ${ }^{26,34,36}$. However, in the present study an inter-electrode distance of $20 \mathrm{~mm}$ was used with a fix location of the surface electrodes over the muscle and the three relationship cases were found.

\section{Limitations}

Limitations to the current research study are acknowledged. First, we did not collect the maximum voluntary isometric contraction (MVIC) from the subjects and thus we used the normalization technique based on peak activation levels obtained during the task under investigation instead. However, this technique can be used to compare patterns of muscle activation between individuals and can decrease the variability between individuals com- pared to using a normalization technique based on MVIC $^{44,57}$. Second, due to the limitation on the number of participants in the present investigation, the analysis of the anthropometric variables should be considered as the feasibility study. However, unlike other tests of significance the ES $d$ is a quantitative measure of the mean different which is independent of sample size ${ }^{46}$. The meaningful anthropometric variables found in the present investigation could be useful in the discrimination of the subjects related to the muscle effect on the MND and MDF methods. Regardless, future research should incorporate a large cohort of subjects from both sexes and across a wide distribution of the human physical variations.

\section{Conclusions}

To assess muscular fatigue during isometric and dynamic muscle contractions, it is necessary to find out the individual effects of all time-varying factors on MNF and MDF of the sEMG signals. Although several time-varying factors have been evaluated in terms of their effects on MNF and MDF during the last three decades, the effect of the muscle's force of contraction is however inconclusive. In this study, three relationship cases have been found: no linear relationship, a positive linear relationship, and a negative linear relationship between the MNF/MDF features and the force of contraction. It is strongly recommended that the levels of muscle contraction and joint angles should be considered together when trying to reduce the muscle effects on MNF and MDF for research purposes. Twelve selected anthropometric variables measured from the subjects were analysed and the results show that it is feasible to use a number of variables as the criteria to create homogeneous subgroups associated with the three relationship cases.

Acknowledgements: This work is jointly funded by Prince of Songkla University and Thailand Research Fund through the Royal Golden Jubilee Ph.D. Programme (Grant no. PHD/0155/2554).

\section{REFERENCES}

1. De Luca CJ (1984) Myoelectrical manifestations of localized muscular fatigue in humans. Crit Rev Biomed Eng 11, 251-79.

2. Al-Mulla MR, Sepulveda F, Colley M (2011) An autonomous wearable system for predicting and detecting localised muscle fatigue. Sensors 11, 1542-57.

3. Al-Mulla MR, Sepulveda F, Colley M (2011) A review of non-invasive techniques to detect and predict localised muscle fatigue. Sensors 11, 3545-94. 
4. Phinyomark A, Phukpattaranont P, Limsakul C (2011) A review of control methods for electric power wheelchairs based on electromyography (EMG) signals with special emphasis on pattern recognition. IETE Tech Rev 28, 316-26.

5. Charoenpanich N, Boonsinsukh R, Sirisup S, Saengsirisuwan V (2013) Principal component analysis identifies major muscles recruited during elite vertical jump. Sci Asia 39, 257-64.

6. Chang KM, Liu SH, Wu XH (2012) A wireless sEMG recording system and its application to muscle fatigue detection. Sensors 12, 489-99.

7. Balasubramanian V, Dutt A, Rai S (2011) Analysis of muscle fatigue in helicopter pilots. Appl Ergon 42, 913-8.

8. Leunkeu AN, Keefer DJ, Imed M, Ahmaidi S (2010) Electromyographic (EMG) analysis of quadriceps muscle fatigue in children with cerebral palsy during a sustained isometric contraction. J Child Neurol 25, 287-93.

9. Oskoei MA, Hu H, Gan JQ (2008) Manifestation of fatigue in myoelectric signals of dynamic contractions produced during playing PC games. In: Proceedings of the 30th Annual International Conference of the IEEE Engineering in Medicine and Biology Society, pp 315-8.

10. Hautier CA, Arsac LM, Deghdegh K, Souquet J, Belli A, Lacour JR (2000) Influence of fatigue on EMG/ force ratio and cocontraction in cycling. Med Sci Sports Exerc 32, 839-43.

11. Cifrek M, Medved V, Tonković S, Ostojić S (2009) Surface EMG based muscle fatigue evaluation in biomechanics. Clin Biomech 24, 327-40.

12. Hendrix CR, Housh TJ, Johnson GO, Mielke M, Camic CL, Zuniga JM, Schmidt RJ (2009) A new EMG frequency-based fatigue threshold test. $J$ Neurosci Meth 181, 45-51.

13. Potvin JR, Bent LR (1997) A validation of techniques using surface EMG signals from dynamic contractions to quantify muscle fatigue during repetitive tasks. J Electromyogr Kinesiol 7, 131-9.

14. Doheny EP, Lowery MM, FitzPatrick DP, O'Malley MJ (2008) Effect of elbow joint angle on force-EMG relationships in human elbow flexor and extensor muscles. J Electromyogr Kinesiol 18, 760-70.

15. Cechetto AD, Parker PA, Scott RN (2001) The effects of four time-varying factors on the mean frequency of a myoelectric signal. $J$ Electromyogr Kinesiol 11, 347-54.

16. De Luca CJ (1979) Physiology, mathematics of myoelectric signals. IEEE Trans Biomed Eng 26, 313-25.

17. Pan ZS, Zhang Y, Parker PA (1989) Motor unit power spectrum and firing rate. Med Biol Eng Comput 27, 14-8.

18. Englehart KB, Parker PA (1994) Single motor unit myoelectric signal analysis with nonstationary data. IEEE Trans Biomed Eng 41, 168-80.
19. Solomonow M, Baten C, Smit J, Baratta R, Hermens H, D'Ambrosia R, Shoji H (1990) Electromyogram power spectra frequencies associated with motor unit recruitment strategies. $J$ Appl Physiol 68, 1177-85.

20. Inbar GF, Allin J, Kranz H (1987) Surface EMG spectral changes with muscle length. Med Biol Eng Comput 25, 683-9.

21. Moritani T, Muramatsu S, Muro M (1987) Activity of motor units during concentric and eccentric contractions. Am J Phys Med 66, 338-50.

22. Shankar S, Gander RE, Brandell BR (1989) Changes in the myoelectric signal (MES) power spectra during dynamic contractions. Electroencephalogr Clin Neurophysiol 73, 142-50.

23. Potvin JR (1997) Effects of muscle kinematics on surface EMG amplitude and frequency during fatiguing dynamic contractions. J Appl Physiol 82, 144-51.

24. Kaplanis PA, Pattichis CS, Hadjileontiadis LJ, Roberts VC (2009) Surface EMG analysis on normal subjects based on isometric voluntary contraction. $J$ Electromyogr Kinesiol 19, 157-71.

25. Viitasalo JT, Komi PV (1978) Interrelationships of EMG signal characteristics at different levels of muscle tension and during fatigue. Electromyogr Clin Neurophysiol 18, 167-78.

26. Petrofsky JS, Lind AR (1980) Frequency analysis of the surface electromyogram during sustained isometric contractions. Eur J Appl Physiol 43, 173-82.

27. Petrofsky JS, Lind AR (1980) The influence of temperature on the amplitude and frequency components of the EMG during brief and sustained isometric contractions. Eur J Appl Physiol 44, 189-200.

28. Hagberg M, Ericsson BE (1982) Myoelectric power spectrum dependence on muscular contraction level of elbow flexors. Eur J Appl Physiol 48, 147-56.

29. Muro M, Nagata A, Murakami K, Moritani T (1982) Surface EMG power spectral analysis of neuromuscular disorders during isometric and isotonic contraction. Am J Phys Med 61, 244-54.

30. Merletti R, Sabbahi MA, De Luca CJ (1984) Median frequency of the myoelectric signal: effects of muscle ischemia and cooling. Eur J Appl Physiol 52, 258-65.

31. Van Boxtel A, Schomaker LRB (1984) Influence of motor unit firing statistics on the median frequency of the EMG power spectrum. Eur J Appl Physiol 52, 207-13.

32. Gander RE, Hudgins RE (1985) Power spectral density of the surface myoelectric signal of the biceps brachii as a function of static load. Electromyogr Clin Neurophysiol 25, 469-78.

33. Inbar GF, Allin J, Paiss O, Kranz H (1986) Monitoring surface EMG spectral changes by the zero crossing rate. Med Biol Eng Comput 24, 10-8.

34. Moritani T, Muro M (1987) Motor unit activity and surface electromyogram power spectrum during increasing force of contraction. Eur J Appl Physiol 56, 260-5. 
35. Hagberg C, Hagberg M (1989) Surface EMG amplitude and frequency dependence on exerted force for the upper trapezius muscle: a comparison between right and left sides. Eur J Appl Physiol 58, 641-5.

36. Gerdle B, Eriksson NE, Brundin L (1990) The behaviour of mean power frequency of the surface electromyogram in biceps brachii with increasing force and during fatigue. With special regard to the electrode distance. Electromyogr Clin Neurophysiol 30, 483-9.

37. Bilodeau M, Arsenault AB, Gravel D, Bourbonnais D (1991) EMG power spectra of elbow extensors during ramp and step isometric contractions. Eur $J$ Appl Physiol 63, 24-8.

38. Rainoldi A, Galardi G, Maderna L, Comi G, Conte LL, Merletti R (1999) Repeatability of surface EMG variables during voluntary isometric contraction of the biceps brachii muscle. J Electromyogr Kinesiol 9, 105-19.

39. Nordander C, Willner J, Hansson G-A, Larsson B, Unge J, Granquist L, Skerfving S (2003) Influence of the subcutaneous fat layer, as measured by ultrasound, skinfold calipers and BMI, on the EMG amplitude. Eur J Appl Physiol 89, 514-9.

40. Stegeman DF, Blok JH, Hermens HJ, Roeleveld K (2000) Surface EMG models: properties and applications. J Electromyogr Kinesiol 10, 313-26.

41. Suuden E, Ereline J, Gapeyeva H, Paasuke M (2008) Low back muscle fatigue during Sorensen endurance test in patients with chronic low back pain: relationship between electromyographic spectral compression and anthropometric characteristics. Electromyogr Clin Neurophysiol 48, 185-92.

42. Thongpanja S, Phinyomark A, Phukpattaranont P, Limsakul C (2013) Mean and median frequency of EMG signal to determine muscle force based on timedependent power spectrum. Elektron Elektrotech 19, 51-6.

43. Phinyomark A, Quaine F, Charbonnier S, Serviere C, Tarpin-Bernard F, Laurillau Y (2013) A feasibility study on the use of anthropometric variables to make muscle-computer interface more practical. Eng Appl Artif Intell 26, 1681-8.

44. Halaki M, Ginn K (2012) Normalization of EMG signals: to normalize or not to normalize and what to normalize to? In: Naik GR (ed) Computational Intelligence in Electromyography Analysis-A Perspective on Current Applications and Future Challenges, InTech, Rijeka, pp 175-94.

45. Taylor R (1990) Interpretation of the correlation coefficient: a basic review. J Diagn Med Sonogr 6, 35-9.

46. Cohen J (1988) Statistical Power Analysis for the Behavioral Sciences, Academic Press, New York.

47. Hof AL (1991) Errors in frequency parameters of EMG power spectra. IEEE Trans Biomed Eng 38, 1077-88.
48. Larivière C, Arsenault AB, Gravel D, Gagnon D, Loisel $P$ (2001) Median frequency of the electromyographic signal: effect of time-window location on brief step contractions. J Electromyogr Kinesiol 11, 65-71.

49. Siegler S, Hillstrom HJ, Freedman W, Moskowitz G (1985) The effect of myoelectric signal processing on the relationship between muscle force and processed EMG. Electromyogr Clin Neurophysiol 25, 499-512.

50. Bilodeau M, Arsenault AB, Gravel D, Bourbonnais D (1992) Influence of gender on the EMG power spectrum during an increasing force level. $J$ Electromyogr Kinesiol 2, 121-9.

51. Deurenberg P, Weststrate JA, Seidell JC (1991) Body mass index as a measure of body fatness: ageand sex-specific prediction formulas. Br J Nutr $\mathbf{6 5}$, 105-14

52. Farina D, Fosci M, Merletti R (2002) Motor unit recruitment strategies investigated by surface EMG variables. J Appl Physiol 92, 235-47.

53. Sabbahi MA, Merletti R, De Luca CJ, Rosenthal RG (1981) How handidness, sex and force level affect the median frequency of the myoelectric signal. In: Proceedings of the 4th Annual Conference on Rehabilitation Engineering, pp 232-4.

54. Polgar J, Johnson MA, Weightman D, Appleton D (1973) Data on fibre size in thirty-six human muscles: an autopsy study. J Neurol Sci 19, 307-18.

55. Komi PV, Viitasalo JHT (1976) Signal characteristics of EMG at different levels of muscle tension. Acta Physiol Scand 96, 267-76.

56. Zipp P (1978) Effect of electrode parameters on the bandwidth of the surface EMG power density spectrum. Med Biol Eng Comput 16, 537-41.

57. Bolgla LA, Uhl TL (2007) Reliability of electromyographic normalization methods for evaluating the hip musculature. J Electromyogr Kinesiol 17, 102-11. 